\title{
Benchmarking pluripotent stem cell-derived organoid models
}

Alejandro De Los Angeles ${ }^{1}$, Elizabeth M. Tunbridge ${ }^{1,2}$

1. Department of Psychiatry, Warneford Hospital, University of Oxford, Oxford, OX3 7JX, United Kingdom.

2. Oxford Health NHS Foundation Trust, Warneford Hospital, University of Oxford, Oxford, OX3 7JX, United Kingdom. 


\begin{abstract}
Cerebral organoids are stem cell-derived, self-organizing three-dimensional cultures. Owing to the remarkable degree to which they recreate the cellular diversity observed in the human brain, they have attracted significant interest as a novel model system for research and drug development, as well as capturing the public imagination. However, many questions remain about the extent to which these cultures recapitulate neurodevelopment and the defining features of the human brain. To clarify the fidelity of human organoid models, Bhaduri and colleagues compared the molecular profile of brain organoid cells with that of primary cells from fetal brain. They observed that, whilst brain organoids broadly recapitulate the cellular profile of human brain, they lack the subtypes of cell classes seen in human brain. In addition, they showed marked expression of cellular stress markers, which could be reversed by transplanting organoid cells into neonatal mouse brain. The authors hypothesise that in vitro culture induces a cellular stress response and that it is this that impairs maturation. Thus, whilst their findings strike a note of caution in the use of organoids as a model for early human brain development, they lay a foundation for improving the accuracy of organoid models in the future.
\end{abstract}




\section{Main Text}

Cerebral organoids are stem cell-derived three-dimensional cultures that model the human brain (Lancaster et al, 2013). Several studies demonstrate that cerebral organoids broadly recapitulate the cellular diversity of the central nervous system (Quadrato et al, 2017; Velasco et al, 2019), making them an attractive platform to dissect human neurobiology. They have therefore been used to study human brain development and evolution, as well as being used as models of brain conditions, including autism spectrum disorder, microcephaly, Miller-Dieker syndrome, Timothy syndrome, and Zika virus infection, among others (Lancaster et al, 2013; Mariani et al, 2015; Garcez et al, 2016; Birey et al, 2017).

While there has been much enthusiasm for the use of organoids to study both healthy brain development and pathophysiological processes, questions remain about the extent to which they accurately model the human brain. Initial data indicate that human pluripotent stem cell-derived organoids model the development of the human fetal cortex at transcriptomic and epigenetic levels (Amiri et al, 2018). However, a systematic benchmarking of organoid models against the developing human brain has been lacking. Therefore, in Nature, Bhaduri and colleagues explored the degree to which human induced pluripotent stem (iPS) cell-derived organoid cells recapitulate characteristics of the developing human brain.

Bhaduri and colleagues first undertook a systematic characterization of the cell types in human fetal cortical primary tissue and organoid samples by single-cell RNA-seq analysis. As anticipated, consistent with earlier reports (Quadrato et al, 2017; Velasco et al, 2019), the authors observed radial glia, intermediate progenitor cells, maturing neurons, and interneurons in both primary human brain tissue and organoids. Importantly, the authors reproducibly generated the repertoire of cell types in organoids using multiple organoid generation protocols and human iPS cell lines (as well as an embryonic stem cell line). Upon closer inspection, however, the authors observed key differences between primary brain and organoid samples. First, while surveying the expression of marker genes, the authors observed multiple, clear subtypes of neural cell classes in brain (e.g. layer IV neuron), the majority of which could not be clearly resolved in organoids. Second, when attempting to distinguish progenitors from neurons, the authors observed co-expression of progenitor and differentiated cell markers, further suggesting incomplete specification of organoid cells. Thus, in primary human brain cells, neurons did not express radial glia markers, and vice-versa, but coexpression of these markers was observed in organoid cells. Finally, organoid radial glia lacked molecular features associated with mature radial glia in primary brain tissue. Taken together, the findings suggest aberrant maturation of neural cells in organoids, compared with human brain.

Neurons from different cortical regions can be distinguished based on their molecular characteristics (Tasic et al, 2018). Bhaduri and colleagues therefore sought to analyze the regional identity of the excitatory neurons present in organoids. They compared the 
molecular characteristics of neurons from defined regions in fetal brain tissue with those from organoids. Surprisingly, individual organoid neurons did possess defined signatures characteristic of specific brain regions; however, examination of key regional markers using immunohistochemistry indicated that neurons with different regional profiles are not clearly segregated. Thus, although organoids contain excitatory neurons with distinct regional identities, these lack the spatial organization seen in human brain. It will be of future interest to determine the regional identity of inhibitory neurons found in stem cell-derived organoids, given evidence for dysfunction of this neuronal class in psychiatric disorders.

A key finding was the observation that gene expression sets related to glycolysis and endoplasmic reticulum (ER) stress were enriched in organoid cells, compared to primary brain cells. Bhaduri and colleagues speculated that these molecular signatures might be inherently related to the in vitro environment. To explore this further, they conducted a series of experiments to investigate the relationships between the culture environment, metabolic stress and cell identity. First, the authors examined the molecular profile of primary cells transplanted into organoids. Consistent with their hypothesis that the in vitro environment induces cellular stress and aberrant maturation, the transplanted cells showed an upregulation of glycolysis and ER stress markers, and developed the 'mixed identity' molecular phenotype (i.e. the lack of clear neural class subtypes observed in organoid cells). Furthermore, these phenotypes were also observed in 3D aggregates of dissociated primary brain cells, suggesting that they result from in vitro aggregate culture, rather than the organoid environment per se. Given these compelling demonstrations of a link between in vitro culture and metabolic stress, the authors investigated whether the cellular stress and 'mixed identity' phenotypes might be rescued by an in vivo environment. They therefore transplanted dissociated organoid cells into neonatal mouse cortex. As predicted, the transplanted cells showed reduced expression of glycolysis and ER stress markers, and improved cell-subtype specification, after five weeks. One possibility is that the transplanted cells that continued to express stress genes had been selected against in the transplanted environment. Regardless, these findings are consistent with the authors' hypothesis that the in vitro environment induces cellular stress, which in turn impairs neural cell subtype specification, as well as indicating that in vivo transplantation might reverse these phenotypes.

Notably, although the authors' findings are consistent with a model whereby in vitro culture induces cellular stress, which in turn impairs the normal maturation of organoid cells, this causal pathway remains to be definitively demonstrated. Furthermore, the molecular mechanisms that might mediate this cascade remain to be elucidated. It is well-established that the expression of different combinations of transcription factors can influence neuronal subtype, and that the overexpression of transcription factors and chromatin modification can even reprogramme neuronal identity (Amamoto and Arlotta, 2014; Lodato et al, 2015). However, the molecular mechanisms that might bridge the gap between changes in glycolysis and ER stress, and cell subtype specification remain 
unknown. Thus, more direct evidence is needed to establish causal links between cellular stress and the aberrant maturation of brain organoids, and to clarify the underlying mechanisms. Such mechanistic insights could inform the development of strategies to reduce culture stress and improve the normal maturation of organoid cells.

An important caveat to the current study is that, consistent with many preceding studies, assessments of cellular maturity were based on gene expression profiling. It will therefore be of future interest to assess the maturity of organoid cells- both in vitro and following transplantation into an in vivo environment - using complementary approaches, including both expanded molecular profiling alongside more functional measures, e.g. morphological and electrophysiological assessments. Such experiments would improve our understanding of maturation within organoids, as well as providing information about how these factors are affected by cellular stress.

Despite the need for additional corroborative evidence, Bhaduri and colleagues' data convincingly demonstrate the active role that the in vivo environment plays in instructing cell identity and maturation. They are consistent with the hypothesis that human-animal interspecies chimeras - animals comprised of cells from human and animal species might be able to overcome some of the limitations associated with conventional 2D differentiation and organoid-based models. For example, it was recently shown that transplantation of human iPS cell-derived microglial precursors into the brains of neonatal mice was correlated with the acquisition of a primary, rather than in vitro cultured, microglia-like state (Svoboda et al, 2019). However, a key limitation with the approach employed in the current manuscript is that it required the dissociation of organoid cells, meaning that human and mouse cells were not spatially separated in the resulting chimera. Whilst well-suited to the study of cell autonomous phenotypes, this 'intermingling' of human and mouse cells may prove problematic for the investigation of phenotypes relying on the presence of intact networks of human neurons and their interactions with other neural cell types.

Altogether, Bhaduri and colleagues' work is a tour de force resource for benchmarking of stem cell-derived organoids against the human brain. Further studies are needed to determine a causative relationship between cellular stress and impaired differentiation, but their findings powerfully illustrates the power of the in vivo environment to improve the maturity of stem cell-derived human neural tissue. Clarification of the mechanisms by which introduction of human cells into chimeric systems promotes cell maturation may, in turn, lead to better in vitro organoid models of human neurodevelopment and pathophysiology. 


\section{References}

Amamoto R, Arlotta P. Development-inspired reprogramming of the mammalian central nervous system. Science 343: 1239882 (2014).

Amiri A, Coppola G, Scuderi S, et al. Transcriptome and epigenome landscape of human cortical development modeled in organoids. Science 362 (6420) (2018).

Bhaduri A, Andrews MG, Mancia Leon W, et al. Cell stress in cortical organoids impairs molecular subtype specification. Nature (2020).

Birey F, Andersen J, Makinson CD, et al. Assembly of functionally integrated human forebrain spheroids. Nature 545: 54 - 59 (2017).

Garcez PP, Loiola EC, Madeiro da Costa R, et al. Zika virus impairs growth in human neurospheres and brain organoids. Science 352: 816 - 8 (2016).

Lancaster MA, Renner M, Martin CA, et al. Cerebral organoids model human brain development and microcephaly. Nature 501: 373 - 9 (2013).

Lodato S, Shetty AS, Arlotta P. Cerebral cortex assembly: generating and reprogramming projection neuron diversity. Trends Neurosci 38: 117 - 25 (2015).

Mariani J, Coppola G, Zhang P, et al. FOXG1-dependent dysregulation of GABA/Glutamate neuron differentiation in autism spectrum disorders. Cell 162: 375 390 (2015).

Quadrato G, Nguyen T, Macosko EZ, et al. Cell diversity and network dynamics in photosensitive human brain organoids. Nature 545: 48 - 53 (2017).

Svoboda DS, Barrasa MI, Shu J, et al. Human iPSC-derived microglia assume a primary microglia-like state after transplantation into the neonatal mouse brain. PNAS 116: 25293-25303 (2019).

Tasic B, et al. Shared and distinct transcriptomic cell types across neocortical areas. Nature 563: 72 - 78 (2018).

Velasco S, Kedaigle AJ, Simmons SK, et al. Individual brain organoids reproducibly form cell diversity of the human cerebral cortex. Nature 570: 523 - 528 (2019). 


\section{Disclosures}

The authors declare no conflicts of interest. 


\section{Acknowledgments}

We thank Paul Harrison for helpful comments on the manuscript. This study was supported by the National Institute for Health Research Oxford Health Biomedical

Research Centre. The views expressed are those of the authors and not necessarily those of the NHS, the NIHR or the Department of Health. 